\title{
Properties of MHD turbulence and its consequences for the ISM and ICM
}

\author{
D. Falceta-Gonçalves ${ }^{1}$, G. Kowal ${ }^{2}$ \& A. Lazarian ${ }^{3}$ \\ ${ }^{1}$ Núcleo de Astrofísica Teórica, Universidade Cruzeiro do Sul - Rua Galvão Bueno 868, CEP \\ 01506-000, São Paulo, Brazil \\ ${ }^{2}$ Jagiellonian University, ul. Orla 171, 30-244 Kraków, Poland \\ ${ }^{3}$ University of Wisconsin, Madison, 475 N. Charter St., WI 53711, USA
}

\begin{abstract}
It is well known that the interstellar (ISM) and intergalactic (ICM) media are threaded by large scale magnetic fields. The understanding of its role on the dynamics of the media is, however, still in progress. For the ISM, magnetic fields may control or, at least, play a major role on the turbulence cascade leading to the star formation process. The ICM, on the other hand, is assumed to be thermally dominated but still the magnetic field may play an important role on the processes of acceleration and propagation of cosmic rays. In this work we provide a review of the latest theoretical results on the evolution of MHD turbulence under collisional and collisionless plasma approaches.
\end{abstract}

Keywords. hydrodynamics, turbulence, methods: numerical, ISM: magnetic fields, galaxies: clusters: general

\section{Turbulence in collisional magnetized plasmas}

Turbulence is, in general, characterized by chaotic fluid motions that result in diffusion of matter and dissipation of kinetic energy. These processes are of great importance, mostly the understanding of how the energy cascades through different scales and gets dissipated in a plasma. In Kolmogorov's theory, large turbulent eddies in an incompressible fluid would fragment to form smaller cells. In this theory, it is assumed that the turbulence is homogeneous, isotropic, scale invariant and local (i.e. waves interact with other waves with similar wavenumber $k$ only). Here, a single physical timescale is defined $\tau_{l} \sim l / \delta v_{l}$, the turnover time, being $l$ the size of the eddy and $v_{l}$ its velocity. The energy transfer rate, assumed to be constant at all scales, will then be $\dot{\epsilon} \sim \delta v_{l}^{2} / \tau_{l}$, and the relations $E(k) \propto k^{-5 / 3}$ and $\delta v_{l} \propto l^{1 / 3}$ are obtained.

The insterstellar medium (ISM), however, is known to be threaded by magnetic fields. In this situation, magnetic perturbations play a role on the energy budget - as new wave modes arise - of the system and change the cascading process of the turbulence. Goldreich \& Sridhar (1995) showed that if wave-wave interactions are strong in magnetized plasmas, i.e. $\tau_{A}=\tau_{s}$, being $\tau_{A}=l / c_{A}$ with $c_{A}$ the Alfven speed, the turbulence is anisotropic $\left(l_{\|} \propto l_{\perp}{ }^{2 / 3}\right)$. The cells are elongated in the magnetic field direction. GS95 model predicted that $E\left(k_{\perp}\right) \propto k_{\perp}{ }^{-5 / 3}$, i.e. alfvenic perturbations are incompressible. Compared to the spectra of the solar wind, which shows almost kolmogorov's cascade but small anisotropy, the answer is that the GS anisotropy occurs at the local magnetic field reference frame. Observations, on the other hand, integrate along the line of sight randomly oriented field lines reducing the observed anisotropy. Obsevations of the ISM have also revealed roughly a Kolmogorov spectrum, as shown in Armostrong, Rickett \& Spangler (1995). Though it is supersonic at most scales (therefore compressible), this scaling can be explained by alfvenic turbulence (see Cho \& Lazarian 2003).

\section{Turbulence in collisionless magnetized Plasmas}

Magnetized and low density (weakly collisional) plasmas are known to present anisotropic pressures with respect to the magnetic field orientation, which can last considerably long compared to the dynamical timescales of certain systems. Under certain conditions, gyrotropic plasmas give rise to new wave modes (see Passot \& Sulem 2006) and instabilities depending on 

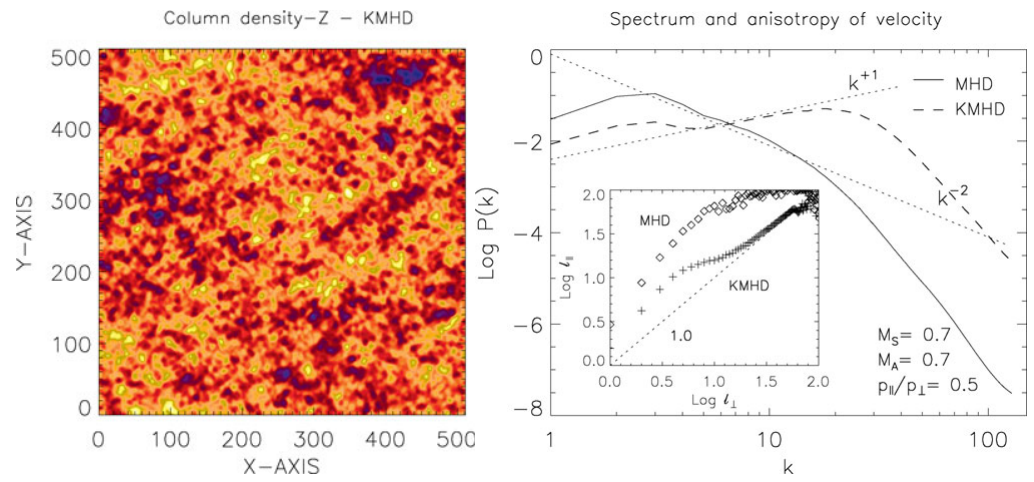

Figure 1. Column density map for the CGL-MHD model with $p_{\|} / p_{\perp}=0.5$, and its spectrum of velocity compared to the collisional MHD case (Falceta-Gonçalves, Kowal \& Lazarian 2009)

the pressure ratio, e.g. the firehose $\left(p_{\|}>p_{\perp}\right)$ and mirror $\left(p_{\perp}>p_{\|}\right)$instabilities, which acts as additional particle accelerating mechanisms and may result in an increase in magnetic field energy. The intracluster medium (ICM) is possibly the most suitable environment for the study of gyrotropic plasma effects.

In this work we simulated the evolution of turbulence in collisionless plasmas, by solving the set of double-isothermal CGL-MHD equations.

In Fig. 1 we present the column density map and spectra of velocity obtained for the CGLMHD model compared, for the model where $p_{\|} / p_{\perp}=0.5$. The CGL-MHD instabilities are responsible for changes in the statistics of the turbulence. Here, the firehose instability is responsible for a curvature of the magnetic field lines. The curved tubes tend to slow down and trap the flowing gas. Since the growth rate is larger at small scales, we expect this effect to create more granulated maps. It is noticeable the power excess at large values of $k$ due to the fast growth of the instabilities in these scales. The slopes of velocity and density spectra for the MHD models range between -1.7 to -2.0 at the inertial range, while CGL-MHD simulations show positive slopes $(\sim+1)$ in velocity spectra, and we must point that we obtain flat density spectra $(\alpha \sim 0)$.

Since ICM plasma is believed to be collisionless, we may expect these intabilities may be present. As a result, comparison with observed spectra, the increase of magnetic field energy and particle acceleration, must be studied in more details.

\section{References}

Armstrong, J. W., Rickett, B. J., \& Spangler, S. R. 1995, ApJ 443, 209

Cho, J. \& Lazarian, A. 2003, MNRAS 345, 325

Falceta-Gonçalves, D., Kowal, G., \& Lazarian, A. 2009, ApJ, submitted

Goldreich, P. \& Sridhar, S. 1995, ApJ 438, 763

Passot, T. \& Sulem, P. L. 2006, J. Geophys. Res., 111, 4203 